\title{
Facile green chemistry approaches towards the synthesis of bis-Schiff bases using ultrasound versus microwave and conventional method without catalyst
}

\author{
Wael A. A. Arafa*a and Raafat M. Shaker ${ }^{\text {b }}$ \\ ${ }^{a}$ Chemistry Department, Faculty of Science, Fayoum University 63514, Fayoum, Egypt \\ ${ }^{b}$ Chemistry Department, Faculty of Science, Minia University, 61519 Minia, Egypt \\ E-mail:waa00@fayoum.edu.eg
}

DOI: $\underline{\text { http://dx.doi.org/10.3998/ark.5550190.p009.464 }}$

\begin{abstract}
A sonochemistry-based method was used to synthesize a novel series of bis-Schiff bases using aromatic aldehydes and diamines (trans-1,4-diaminocyclohexane, p-xylylenediamine and ethylenediamine dihydrochloride) without catalyst. Yields and reaction times needed for reaction completion using conventional heating, microwave (MW) and ultrasound (US) irradiation are compared. The environmentally friendly sonochemical waves, in the presence of electron withdrawing and electron donating groups, afford the desired products in high yields and short time. The structures of the products were proven by elemental analyses, IR, MS, ${ }^{1} \mathrm{H},{ }^{19} \mathrm{~F}$, and ${ }^{13} \mathrm{C}$ NMR spectroscopy. ${ }^{1} \mathrm{H}$ NMR spectral data revealed that some derivatives have stronger intramolecular hydrogen bonding than others.
\end{abstract}

Keywords: Bis-Schiff bases, ultrasound irradiation, conventional method, microwave irradiation

\section{Introduction}

Synthesis and application of Schiff bases have been highly considered in recent decades. ${ }^{1,2}$ Schiff bases with its azomethine functional group $(-\mathrm{CH}=\mathrm{N}-)$ are reported to show a wide range of pharmacological activities. ${ }^{3,4}$ They have been reported to exhibit antimicrobial, ${ }^{5,6}$ antibacterial, ${ }^{7,8}$ anti-inflammatory, ${ }^{9}$ antimalarial, ${ }^{10}$ antioxidant, ${ }^{11,12}$ antiproliferative, ${ }^{12}$ antiviral ${ }^{13}$ antipyretic, ${ }^{14}$ antifungal, ${ }^{13}$ antitumor, ${ }^{15}$ analgesic, ${ }^{16}$ anticonvulsant, ${ }^{17,18}$ urease inhibitory, ${ }^{19}$ and anticancer ${ }^{20}$ activities. Also, the structural activity relationship of Schiff bases have been studied worldwide as it is proven that the $-\mathrm{N}=\mathrm{CH}$ - linkage in Schiff bases is an essential feature for bioactivity. ${ }^{21,22}$ One of the most interesting structural features of Schiff bases, which have been prepared from aromatic ortho-hydroxy aldehydes, is the presence of intramolecular hydrogen bonding between the $\mathrm{OH}$ hydrogen and $\mathrm{C}=\mathrm{N}$ nitrogen atoms, ${ }^{23}$ which determine the properties of various 
molecular systems and play a significant role in many biochemical mechanisms. ${ }^{24}$ As well, the intramolecular proton transfer equilibrium is known to be crucial for physicochemical properties and practical application of Schiff bases and this process has been widely studied. ${ }^{25}$ In addition, the synthesis of bis-Schiff bases has been attracting increasing interest in a number of areas in biochemistry as well as chemistry. ${ }^{26}$ Symmetrical bis-Schiff bases have been studied due to their pronounced pharmacological and biological activities, ${ }^{27}$ optical, ${ }^{28}$ photochromical ${ }^{29}$ and thermochromical $^{30}$ properties. They have also been used in the design of liquid crystal materials, ${ }^{31}$ as the building blocks for the preparation of oligomers or liquid crystal polymers ${ }^{32}$ and for the synthesis of organic thin-film transistors. ${ }^{33}$

Recently, the application of ultrasound as a powerful technique in synthetic organic chemistry has become extremely efficient and attractive. Prominent features of the ultrasound approach are enhanced organic reaction rates, formation of purer products in high yields and under mildes reaction conditions. Further, it is considered a processing aid in terms of energy conservation and waste minimization compared to traditional methods. ${ }^{34,35}$ Prompted by the aforementioned biological and pharmaceutical activities, and as a part of an ongoing program aiming at the synthesis of bis-heterocyclic compounds ${ }^{36-38}$ and preparation of medicinally significant structures, ${ }^{39,40}$ we describe herein an efficient and direct procedure for the synthesis of a novel series of bis-Schiff bases, using ultrasound irradiation without catalyst.

\section{Results and Discussion}

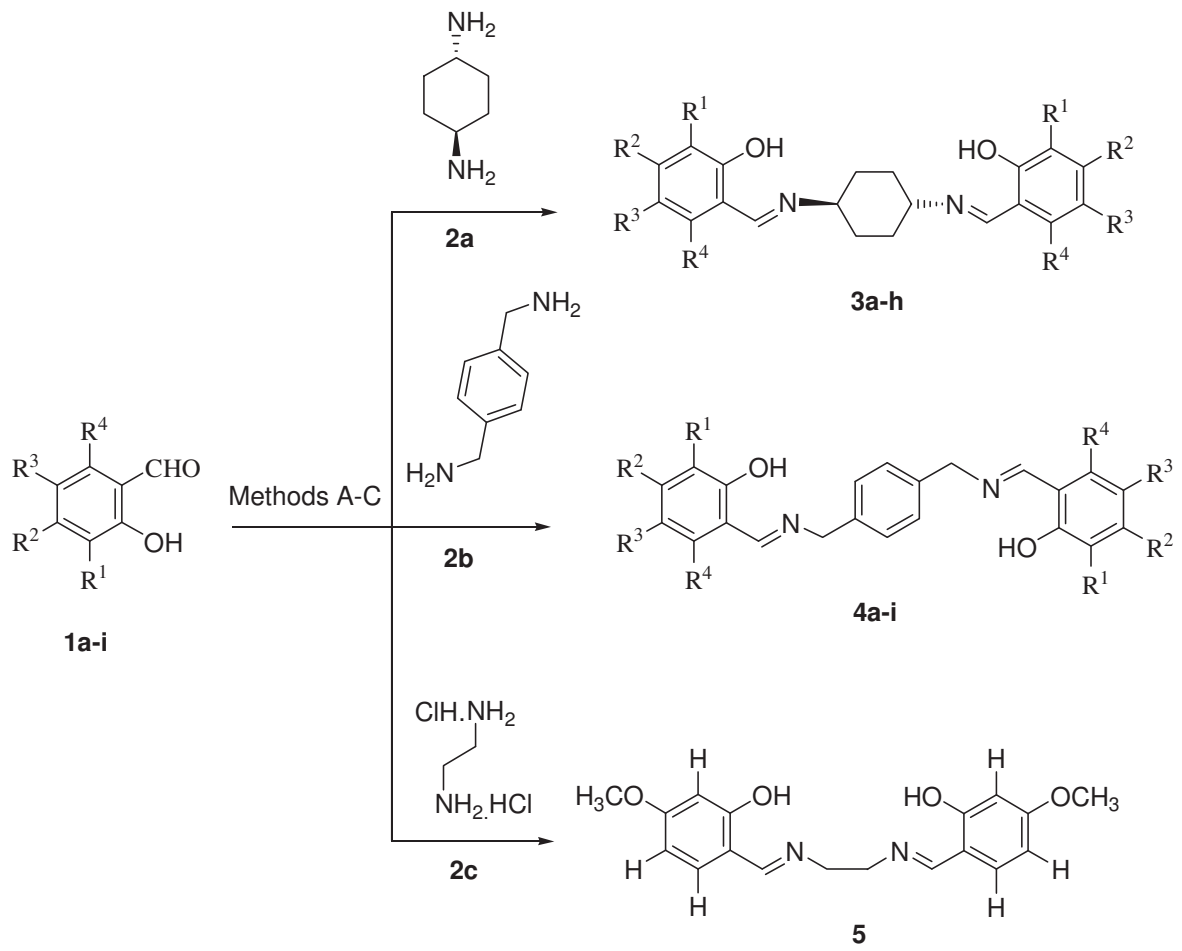

Scheme 1. Synthesis of bis-Schiff bases 3-5. Reaction conditions: Method A, US: EtOH, rt, 1-4 min.; Method B, Convn.: EtOH, rt, 10-12h; Method C, MW: 6-10 min. 
Scheme 1 outlines the synthesis of bis-Schiff bases 3-5 from the reaction of the salicylaldehyde derivatives $\mathbf{1 a - i}$ with diamines 2a-c using ultrasound irradiation as well as conventional synthetic and microwave irradiation methods (Table 1).

Table 1. Formation of compounds 3-5 by (conv. = conventional, MW = microwave irradiation and US = ultrasound)

\begin{tabular}{|c|c|c|c|c|c|c|c|c|c|c|}
\hline \multirow{2}{*}{$\begin{array}{c}\text { Compd. } \\
\text { No. }\end{array}$} & \multirow{2}{*}{$\mathbf{R}^{1}$} & \multirow{2}{*}{$\mathbf{R}^{2}$} & \multirow{2}{*}{$\mathbf{R}^{3}$} & \multirow{2}{*}{$\mathbf{R}^{4}$} & \multicolumn{3}{|c|}{ Time (min.) } & \multicolumn{3}{|c|}{ Yield (\%) } \\
\hline & & & & & US & conv. & MW & US & conv. & MW \\
\hline 3a & $\mathrm{H}$ & $\mathrm{H}$ & $\mathrm{CH}_{3}$ & $\mathrm{H}$ & 2 & 660 & 6 & 99 & 86 & 85 \\
\hline $3 b$ & $\mathrm{H}$ & $\mathrm{H}$ & $\mathrm{OCH}_{3}$ & $\mathrm{H}$ & 2 & 660 & 6 & 99 & 86 & 87 \\
\hline $3 c$ & $\mathrm{H}$ & $\mathrm{OCH}_{3}$ & $\mathrm{H}$ & $\mathrm{H}$ & 2 & 660 & 6 & 99 & 85 & 87 \\
\hline $3 d$ & $\mathrm{C}\left(\mathrm{CH}_{3}\right)_{3}$ & $\mathrm{H}$ & $\mathrm{C}\left(\mathrm{CH}_{3}\right)_{3}$ & $\mathrm{H}$ & 2 & 600 & 10 & 99 & $92(97)^{41}$ & 78 \\
\hline $3 e$ & $\mathrm{H}$ & $\mathrm{H}$ & $\mathrm{NO}_{2}$ & $\mathrm{H}$ & 4 & 720 & 8 & 97 & 76 & 80 \\
\hline $3 f$ & $\mathrm{H}$ & $\mathrm{H}$ & \multicolumn{2}{|c|}{$\mathrm{CH}=\mathrm{CHCH}=\mathrm{CH}$} & 2 & 660 & 10 & 99 & 82 & 82 \\
\hline $3 g$ & $\mathrm{H}$ & $\mathrm{H}$ & $\mathrm{F}$ & $\mathrm{H}$ & 2 & 720 & 10 & 99 & 77 & 79 \\
\hline $3 \mathbf{h}$ & $\mathrm{H}$ & $\mathrm{H}$ & $\mathrm{Br}$ & $\mathrm{H}$ & 2 & 720 & 10 & 98 & 72 & 76 \\
\hline $4 a$ & $\mathrm{H}$ & $\mathrm{H}$ & $\mathrm{H}$ & $\mathrm{H}$ & 2 & 720 & 10 & 99 & $80^{42}$ & 88 \\
\hline $4 b$ & $\mathrm{H}$ & $\mathrm{H}$ & $\mathrm{CH}_{3}$ & $\mathrm{H}$ & 2 & 720 & 10 & 99 & 76 & 80 \\
\hline $4 c$ & $\mathrm{H}$ & $\mathrm{H}$ & $\mathrm{OCH}_{3}$ & $\mathrm{H}$ & 2 & 720 & 10 & 99 & 80 & 80 \\
\hline $4 d$ & $\mathrm{H}$ & $\mathrm{OCH}_{3}$ & $\mathrm{H}$ & $\mathrm{H}$ & 2 & 600 & 10 & 99 & 81 & 80 \\
\hline $4 e$ & $\mathrm{C}\left(\mathrm{CH}_{3}\right)_{3}$ & $\mathrm{H}$ & $\mathrm{C}\left(\mathrm{CH}_{3}\right)_{3}$ & $\mathrm{H}$ & 2 & 660 & 10 & 99 & $86(88)^{43}$ & 79 \\
\hline $4 f$ & $\mathrm{H}$ & $\mathrm{H}$ & $\mathrm{NO}_{2}$ & $\mathrm{H}$ & 2 & 720 & 10 & 99 & 70 & 74 \\
\hline $4 \mathrm{~g}$ & $\mathrm{H}$ & $\mathrm{H}$ & \multicolumn{2}{|c|}{$\mathrm{CH}=\mathrm{CHCH}=\mathrm{CH}$} & 2 & 720 & 10 & 99 & 76 & 78 \\
\hline $4 h$ & $\mathrm{H}$ & $\mathrm{H}$ & $\mathrm{F}$ & $\mathrm{H}$ & 2 & 660 & 10 & 99 & 83 & 82 \\
\hline $4 \mathbf{i}$ & $\mathrm{H}$ & $\mathrm{H}$ & $\mathrm{Br}$ & $\mathrm{H}$ & 2 & 720 & 10 & 99 & 67 & 70 \\
\hline 5 & $\mathrm{H}$ & $\mathrm{OCH}_{3}$ & $\mathrm{H}$ & $\mathrm{H}$ & 1 & 720 & 10 & 99 & $80(80)^{44}$ & 88 \\
\hline
\end{tabular}

In a preparatory experiment, the synthesis of bis-Schiff bases 3-5 was achieved by the reaction of salicylaldehydes $\mathbf{1 a - i}(2 \mathrm{mmol})$ and diamines $\mathbf{2 a - c}(1 \mathrm{mmol})$ in ethanol at room temperature $\left(25^{\circ} \mathrm{C}\right)$. After stirring for $10-12 \mathrm{~h}$, the obtained solid was isolated to give bis-Schiff bases 3-5 in 67-92 \% yield (Table 1). To further improve the yield and decrease the reaction time, the above reaction was carried out under microwave irradiation but there is no valuable improvement in the reaction yield (70-88\%) (Table 1). The yield of the microwave-assisted protocol not increased even when very long reaction times were used.

A potential method for the synthesis of bis-Schiff base compounds 3-5 was achieved by mixing different salicylaldehyde derivatives 1a-i and diamines 2a-c in a molar ratio of 2:1, respectively in ethanol and the reaction mixture was exposed to ultrasound irradiation for 1-4 min (reaction complete based on TLC analysis) (Table 1). The crude reaction mixture was 
examined by ${ }^{1} \mathrm{H}$ NMR spectroscopy which indicated the presence of only one major product. The reaction using ultrasound irradiation leads to an isolated yield of $>97 \%$ (Table 1). Advantages of this efficient method are time-saving, excellent yield of products in pure form, and the simplicity of the work up procedure. A comparison of this ultrasound-based synthetic approach with conventional synthetic or microwave irradiation methods demonstrates that our new methodology is robust and compatible with electron donating and electron withdrawing groups affording the desired products in high yields in just a couple of minutes vs. hours when using conventional method. The non-conventional energy source of ultrasound demonstrates its superiority, in terms of yield, reaction time and operational simplicity. This result is due to the phenomenon of acoustic cavitation, which leads to many unique properties such as creating, enlarging and imploding gaseous and vaporous cavities in the irradiated liquid. ${ }^{45}$ Thus, under sonication, the reaction mixture is activated by inducing a high local temperature and pressure generation inside this cavitation bubble at its interfaces when it collapses, speeding up the reaction and leading to shorter reaction times. Analytical and spectroscopic data for the compounds 3-5 are given in the experimental section and agree well with the expected values. IR spectra of bis-Schiff bases 3-5 showed the principal band between $1623-1607 \mathrm{~cm}^{-1}$ assigned to the double bond stretching of a azomethine function $(\mathrm{C}=\mathrm{N})$ conjugated to an aromatic ring. Furthermore, the appearance of a broad medium strong band around $3287-3264 \mathrm{~cm}^{-1}$ can be ascribed to the existence of intramolecular hydrogen bond of the ortho $\mathrm{OH}$ groups with $\mathrm{N}=\mathrm{CH}$. The NMR spectra of compounds 3-5 showed chemical shifts, which are characteristics for the anticipated structure. For example, the bis-Schiff bases, 3-5, ${ }^{1} \mathrm{H}$ NMR showed that the proton attached to the oxygen atom (OH group) is very acid because it appeared between 14.80-12.77 ppm; this is probably due to the intramolecular hydrogen bonding. It is known that hydrogen bonding shifts the resonance signal of a proton to higher frequency (lower field). Comparing the ${ }^{1} \mathrm{H}$ NMR data of $\mathrm{OH}$ protons, it can be said that the strongest intramolecular hydrogen bond $(\mathrm{N} \cdots \mathrm{OH})$ is formed in $\mathbf{3 f}(\delta 14.80 \mathrm{ppm}, \mathrm{OH})$ (Figure 1), and the weakest one in $\mathbf{4 d}(\delta 12.77 \mathrm{ppm}$, $\mathrm{OH})$. It is interesting that $12.77 \mathrm{ppm}$ value of the compound $\mathbf{4 d}$ is lower than those of the other phenolic Schiff bases. ${ }^{46}$

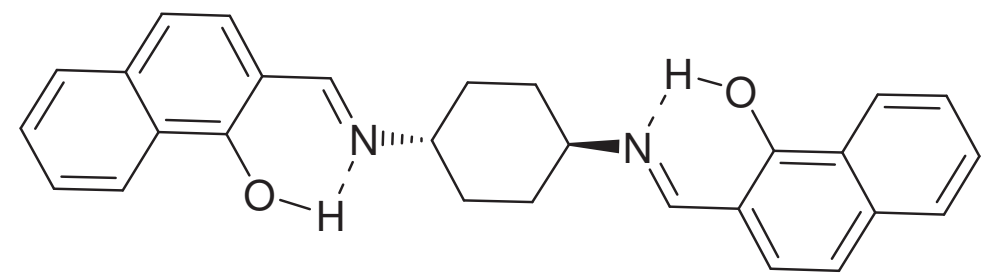

Figure 1. Intramolecular hydrogen bonding at compound $\mathbf{3 f}$.

This difference means that the phenolic $\mathrm{OH}$ proton of $\mathbf{4 d}$ has less acidic character and, consequently, the compound $\mathbf{4 d}$ has weaker intramolecular hydrogen bonding comparing with the others. Reason of this might be due to compound $4 \mathbf{d}$ has keto-enol tautomers (Figure 2). A 
similar situation was observed from the melting points of these bis-Schiff bases: melting point of $\mathbf{4 d}$ is lower than those of the other compounds (See experimental section).
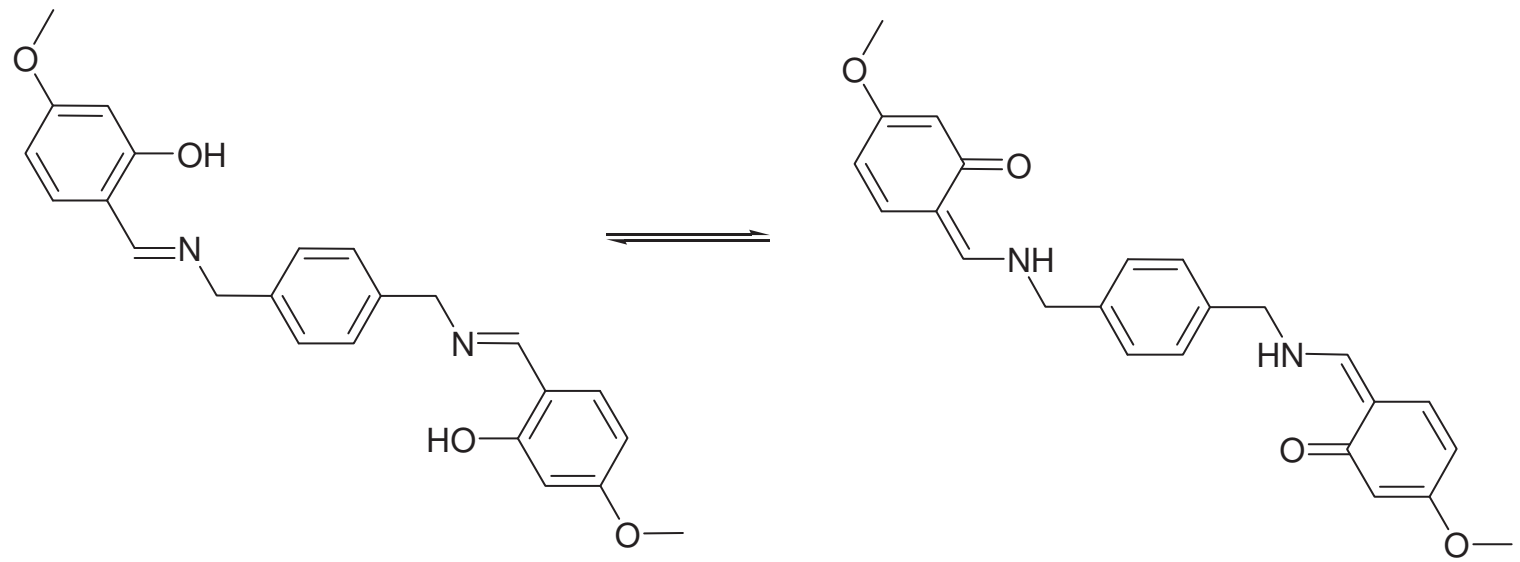

Figure 2. Keto-enol tautomerism at compound $\mathbf{4 d}$.

Moreover, the signal of the proton of the CHO group disappeared in all ${ }^{1} \mathrm{H}$ NMR spectra that confirm the formation of Schiff bases. The singlets observed between 8.92 and $8.18 \mathrm{ppm}$ are assigned to the azomethine $(\mathrm{CH}=\mathrm{N})$ protons. Additionally, for compounds $\mathbf{3 a}-\mathbf{h}$, the protons of the cyclohexane ring are shown in the $3.65-1.66 \mathrm{ppm}$ range as multiples in almost derivatives. For $\mathbf{4 a - i}$, the protons of the disubstituted benzene ring are shown in the 7.44-8.28 ppm range as singlets and for compound $\mathbf{5}$, the protons of the ethylene part are shown at $3.82 \mathrm{ppm}$ as a singlet. In the ${ }^{13} \mathrm{C}$ NMR spectra of the bis-Schiff bases 3-5, the carbon atoms of the azomethine groups were shown in the 166.7-158.3 ppm range. Moreover, the structures of all bis-Schiff bases 3-5 were further confirmed by mass spectra (MS) and elemental analyses.

Interestingly, the newly synthesized bulky bipyridine- $N$-oxide $\mathbf{8}$ also reacted with diamines 2a-c under the same reaction conditions to provide the corresponding bis-Schiff bases 9a-c in excellent yield (Table 2). The bipyridine- $N^{\prime}$-oxide $\mathbf{8}$ was obtained from the reaction of 5-ethynyl2-hydroxybenzaldehyde 6 with 4'-azido-2,2'-bipyridine- $N$ '-oxide 7 (Scheme 2). The NMR and IR spectra, as well as the MS of compounds $\mathbf{8}$ and 9a-c were in agreement with the proposed structures. For example, the ${ }^{1} \mathrm{H}$ NMR spectrum of $\mathbf{8}$ in $\mathrm{CDCl}_{3}$ exhibited two sharp singlets readily recognized as arising from the aldehyde $(\delta 10.02 \mathrm{ppm})$ and hydroxyl protons $(\delta 11.13$ $\mathrm{ppm})$. The ${ }^{13} \mathrm{C}$ NMR spectrum of $\mathbf{8}$ exhibited 18 signals in agreement with the proposed structure. The MS analyses of derivatives 9a-c revealed that it contained two moles of bipyridine- $N$ '-oxide 8 per mole of diamine 2a-c. Moreover, the ${ }^{1} \mathrm{H}$ NMR spectra of 9a-c showed two singlet resonances between 13.92-13.70 and 9.44-9.42 ppm assigned to the phenolic (OH) and azomethine $(\mathrm{CH}=\mathrm{N})$ protons, respectively. Also, IR spectroscopy confirmed the presence of the azomethine groups. 
<smiles>C#Cc1ccc(O)c(C=O)c1</smiles>

6<smiles></smiles>

7<smiles>O=Cc1cc(-c2cn(-c3cc[n+]([O-])c(-c4ccccn4)c3)nn2)ccc1O</smiles>

8
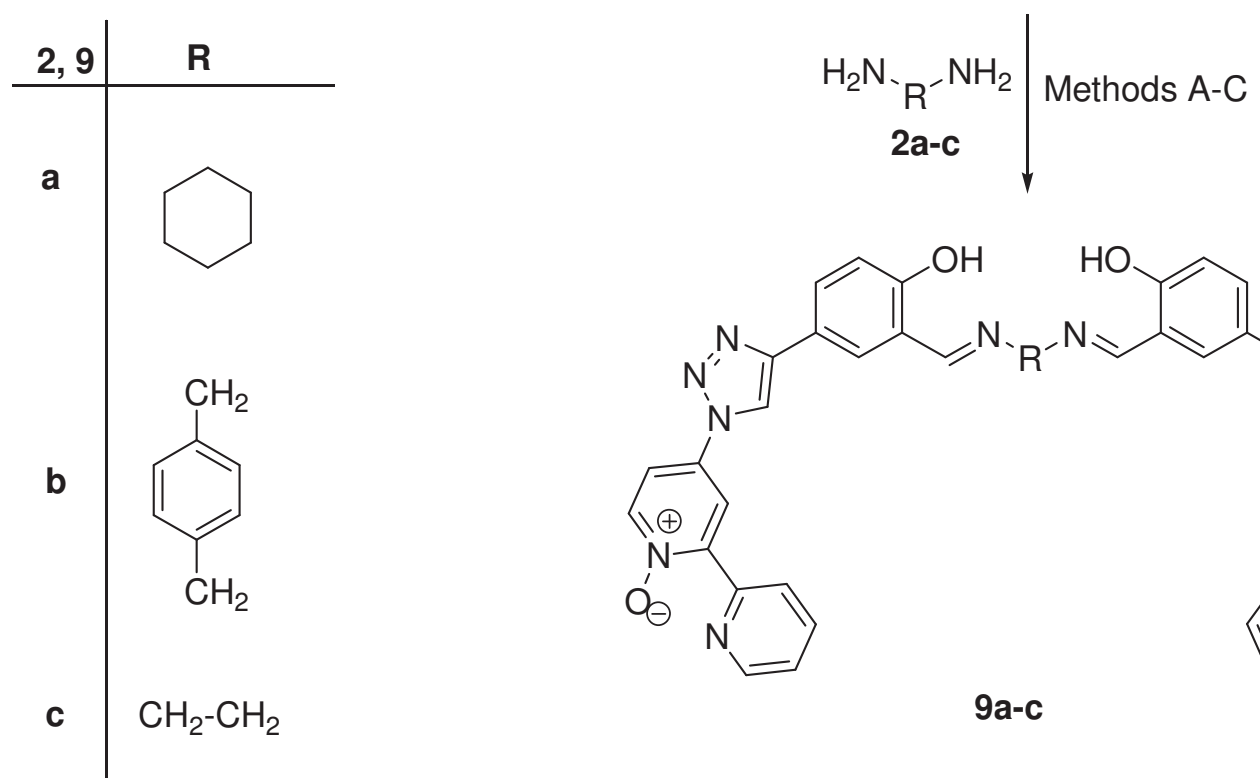

Scheme 2. Synthesis of bis-Schiff bases 9a-c.

Table 2. Formation of compounds 9a-c by (conv. = conventional, MW = microwave irradiation and US = ultrasound)

\begin{tabular}{|c|c|c|c|c|c|c|c|}
\hline \multirow{2}{*}{$\begin{array}{c}\text { Compd } \\
\text { No. }\end{array}$} & \multirow{2}{*}{$\mathbf{R}$} & \multicolumn{3}{|c|}{ Time (min.) } & \multicolumn{3}{|c|}{ Yield (\%) } \\
\hline & & US & conv. & MW & US & conv. & MW \\
\hline 9a & & 4 & 720 & 10 & 95 & 65 & 71 \\
\hline $9 b$ & & 4 & 720 & 10 & 96 & 55 & 60 \\
\hline $9 c$ & $\mathrm{CH}_{2} \mathrm{CH}_{2}$ & 3 & 720 & 10 & 99 & 67 & 76 \\
\hline
\end{tabular}




\section{Conclusions}

In this paper, we described a significant protocol to synthesize a series of novel bis-Schiff bases under sonication, microwave and conventional methods without catalyst. It was observed that, the use of ultrasound improved the yield and the rate of the reaction. The structures of all the synthesized compounds have been confirmed by IR, NMR, MS and elemental analyses.

\section{Experimental Section}

Materials. The solvents and organic reagents (aldehyde derivatives, $p$-xylylenediamine, ethylenediamine dihydrochloride (ethylenediamine dihydrochloride was neutralized by sodium carbonate solution (10\%) before use) and trans-1,4-diaminocyclohexane) required for the synthesis of bis-Schiff bases were purchased from Sigma-Aldrich and used without further purification. The purity of all the compounds was routinely checked by TLC on Silica gel-GF 254 (Merck) coated plates. 5-Ethynyl-2-hydroxybenzaldehyde (6) ${ }^{47}$ and 4-azido-2,2'-bipyridine$N$-oxide $(7)^{48}$ were synthesized according to procedures described in the literature.

Apparatus. The melting points of the compounds were determined on Electrothermal IA9100 melting point apparatus (UK). Mass spectra measurements were recorded on a Bruker Daltonics microTOF spectrometer with an electrospray ionizer. IR spectra were recorded on a PerkinElmer Spectrum One spectrometer, using samples prepared as $\mathrm{KBr}$ discs. ${ }^{1} \mathrm{H},{ }^{13} \mathrm{C}$ and ${ }^{19} \mathrm{~F}$ NMR spectra were recorded at 400,100 and $376 \mathrm{MHz}$, respectively. Chemical shifts $(\delta)$ are reported in ppm, using the residual solvent $\mathrm{CDCl}_{3} \delta(\mathrm{H})=7.26$ and $\delta(\mathrm{C})=77.16$; DMSO- $d_{6} \delta(\mathrm{H})=2.50$ and $\delta(C)=39.52)$ as internal standard. Splitting patterns are designated as singlet (s), doublet (d), triplet $(\mathrm{t})$, doublet of doublets $(\mathrm{dd})$, doublet of triplets $(\mathrm{dt})$, triplet of doublets (td), broad (br). Splitting patterns that could not be interpreted or easily visualized are designated as multiplets (m). Elemental analyses were carried out at MEDAC Ltd, Chobham, Surrey, United Kingdom. Microwave irradiation was carried out using Biotage ${ }^{\circledR}$ Initiator Classic (Biotage AB; Uppsala, Sweden) using sealed vessels. Ultrasonic reactions were carried out in Clifton Ultrasonic Bath (2 x T2A, 300 W, DU-4) made by Nickel Electro Ltd, Weston-super-Mare, Somerset, England.

\section{General procedures for the synthesis of compounds 3-5}

Conventional method. Salicylaldehyde derivatives $\mathbf{1 a - i}(2 \mathrm{mmol})$ and diamines $\mathbf{2 a - c}(1 \mathrm{mmol})$ were suspended in ethanol $(5 \mathrm{~mL})$. The reaction mixture was stirred for $10-12 \mathrm{~h}$ at room temperature $\left(25^{\circ} \mathrm{C}\right)$. The reaction was monitored on TLC. The resulting precipitate is filtered and washed with ethanol/water mixture $(3 \times 10 \mathrm{~mL})$ to afford pure desired product (see Table 1$)$.

Microwave method. Salicylaldehyde derivatives 1a-i $(2 \mathrm{mmol})$ and diamines $\mathbf{2 a - c}(1 \mathrm{mmol})$ were mixed thoroughly and irradiated at $450 \mathrm{~W}$ for $6-10 \mathrm{~min}$ at room temperature $\left(25{ }^{\circ} \mathrm{C}\right)$. The reaction mixture was taken in dichloromethane $(\mathrm{DCM})(20 \mathrm{~mL})$ and washed with water $(3 \times 5$ 
$\mathrm{mL}$ ). The DCM layer was dried over anhydrous magnesium sulfate. Removal of DCM under reduced pressure gave pure compound (see Table 1).

Ultrasonication method. A reaction flask containing salicylaldehyde derivatives 1a-i ( $2 \mathrm{mmol})$, diamines 2a-c $(1 \mathrm{mmol})$ and $5 \mathrm{~mL}$ absolute ethanol was immersed in an ultrasonic bath containing water at room temperature $\left(25^{\circ} \mathrm{C}\right)$. The reaction mixture was exposed to ultrasound irradiation for 1-4 min (reaction complete based on TLC analysis). The resulting precipitate is filtered and washed with ethanol/water mixture $(3 \times 10 \mathrm{~mL})$ to afford the pure desired product (see Table 1).

$\boldsymbol{N}, \boldsymbol{N}^{\prime}$-Bis-(5-methylsalicylidene)--trans-(1,4-cyclohexylenediamine) (3a). yellow solid, $\mathrm{mp}$ 215-216 ${ }^{\circ} \mathrm{C}$. ${ }^{1} \mathrm{H}$ NMR (400 MHz, $\mathrm{CDCl}_{3}$ ): $\delta_{\mathrm{H}} 13.28$ (s, 2H), 8.37 (s, 2H), $7.13-7.10$ (m, 2H), $7.05(\mathrm{~d}, J 2.0 \mathrm{~Hz}, 2 \mathrm{H}), 6.87(\mathrm{~s}, 1 \mathrm{H}), 6.85(\mathrm{~s}, 1 \mathrm{H}), 3.28(\mathrm{dd}, J$ 6.7, $3.4 \mathrm{~Hz}, 2 \mathrm{H}), 2.29(\mathrm{~s}, 6 \mathrm{H}), 1.97$ - $1.95(\mathrm{~m}, 4 \mathrm{H}), 1.74-1.69(\mathrm{~m}, 4 \mathrm{H}) .{ }^{13} \mathrm{C} \mathrm{NMR}\left(100 \mathrm{MHz}, \mathrm{CDCl}_{3}\right): \delta_{\mathrm{C}} 163.0(2 \mathrm{C}, \mathrm{CH}=\mathrm{N}), 158.8$, 132.9, 131.2, 127.6, 118.5, 116.7 (12C, Ar), 66.9, 32.3 (6C, Cyclohexane), 20.3 (2C, $\left.\mathrm{CH}_{3}\right)$. IR $\left(\mathrm{KBr}, v_{\max }, \mathrm{cm}^{-1}\right): 3269(\mathrm{OH})$ and $1608(\mathrm{C}=\mathrm{N})$. MS (EI): $\mathrm{m} / z, 351(\mathrm{M}+\mathrm{H})^{+}, 373(\mathrm{M}+\mathrm{Na})^{+}$. Anal. calcd. for $\mathrm{C}_{22} \mathrm{H}_{26} \mathrm{~N}_{2} \mathrm{O}_{2}$ : C, 75.40; H, 7.48; N, 7.99\%. Found: C, 75.38; H, 7.50; N, 7.95\%.

$\boldsymbol{N}, \boldsymbol{N}^{\prime}$-Bis-(5-methoxysalicylidene)--trans-(1,4-cyclohexylenediamine) (3b). Yellow solid, $\mathrm{mp}$ 247-248 ${ }^{\circ} \mathrm{C} ;{ }^{1} \mathrm{H}$ NMR (400 MHz, $\mathrm{CDCl}_{3}$ ): $\delta_{\mathrm{H}} 13.02$ (s, 2H), 8.41 (s, 2H), $7.28(\mathrm{~s}, 1 \mathrm{H}), 6.94-6.93$ $(\mathrm{m}, 3 \mathrm{H}), 6.81(\mathrm{~d}, J 2.5 \mathrm{~Hz}, 2 \mathrm{H}), 3.81(\mathrm{~s}, 6 \mathrm{H}), 3.31(\mathrm{br}, 2 \mathrm{H}), 2.01-1.99(\mathrm{~m}, 4 \mathrm{H}), 1.77$ - $1.72(\mathrm{~m}$, $4 \mathrm{H}) .{ }^{13} \mathrm{C} \mathrm{NMR}\left(100 \mathrm{MHz}, \mathrm{CDCl}_{3}\right): \delta_{\mathrm{C}} 162.8(2 \mathrm{C}, \mathrm{CH}=\mathrm{N}), 155.1,152.0,119.1,118.5,117.6$, 114.8 (12C, Ar), 67.0 (2C, Cyclohexane), 55.9 (2C, $\left.\mathrm{OCH}_{3}\right), 32.2$ (4C, Cyclohexane). IR ( $\mathrm{KBr}$, $\left.v_{\max }, \mathrm{cm}^{-1}\right): 3271(\mathrm{OH})$ and $1612(\mathrm{C}=\mathrm{N})$. MS $(\mathrm{EI}): \mathrm{m} / z 383(\mathrm{M}+\mathrm{H})^{+}$. Anal. calcd. for $\mathrm{C}_{22} \mathrm{H}_{26} \mathrm{~N}_{2} \mathrm{O}_{4}$ : C, 69.09; H, 6.85; N, 7.32\%. Found: C, 69.12; H, 6.77; N, 7.33\%.

$\boldsymbol{N}, \boldsymbol{N}^{\prime}$-Bis-(4-methoxysalicylidene)-trans-(1,4-cyclohexylenediamine) (3c). yellow solid, $\mathrm{mp}$ 259-260 ${ }^{\circ} \mathrm{C} .{ }^{1} \mathrm{H}$ NMR (400 MHz, $\mathrm{CDCl}_{3}$ ): $\delta_{\mathrm{H}} 14.03$ (s, 2H), 8.25 (s, 2H), $7.11(\mathrm{~s}, 1 \mathrm{H}), 7.09$ (s, $1 \mathrm{H}), 6.42-6.37(\mathrm{~m}, 4 \mathrm{H}), 3.81(\mathrm{~s}, 6 \mathrm{H}), 3.28-3.26(\mathrm{~m}, 2 \mathrm{H}), 2.00-1.97(\mathrm{~m}, 4 \mathrm{H}), 1.71-1.66(\mathrm{~m}$, 4H). ${ }^{13} \mathrm{C}$ NMR (100 MHz, $\left.\mathrm{CDCl}_{3}\right) ; \delta_{\mathrm{C}} 165.6,163.6(4 \mathrm{C}, \mathrm{Ar}), 162.0(2 \mathrm{C}, \mathrm{CH}=\mathrm{N}), 132.5,112.3$, 106.3, 101.2 (8C, Ar), 65.2 (2C, Cyclohexane), 55.3 (2C, $\left.\mathrm{OCH}_{3}\right), 32.2$ (4C, Cyclohexane). IR $\left(\mathrm{KBr}, v_{\max }, \mathrm{cm}^{-1}\right): 3270(\mathrm{OH})$ and $1609(\mathrm{C}=\mathrm{N})$. MS $(\mathrm{EI}): \mathrm{m} / z 383(\mathrm{M}+\mathrm{H})^{+}$. Anal. calcd. for $\mathrm{C}_{22} \mathrm{H}_{26} \mathrm{~N}_{2} \mathrm{O}_{4}$ : C, 69.09; H, 6.85; N, 7.32\%. Found: C, 69.11; H, 6.79; N, 7.40\%.

$\boldsymbol{N}, \boldsymbol{N}^{\prime}$-Bis-(3,5-di-tert-butylsalicylidene)-trans-(1,4-cyclohexylenediamine) $\quad(3 \mathrm{~d}) .{ }^{41} \quad$ Yellow solid, mp 302-303 ${ }^{\circ} \mathrm{C} .{ }^{1} \mathrm{H}$ NMR (400 MHz, $\mathrm{CDCl}_{3}$ ): $\delta_{\mathrm{H}} 13.86$ (s, 2H), 8.44 (s, 2H), 7.39 (dd, J 6.5, $2.5 \mathrm{~Hz}, 2 \mathrm{H}), 7.11(\mathrm{~d}, J 2.4 \mathrm{~Hz}, 2 \mathrm{H}), 3.28-3.27$ (m, 2H), $1.99-1.96(\mathrm{~m}, 4 \mathrm{H}), 1.76-1.72(\mathrm{~m}$, $4 \mathrm{H}), 1.46(\mathrm{~s}, 18 \mathrm{H}), 1.31(\mathrm{~s}, 18 \mathrm{H}) \cdot{ }^{13} \mathrm{C} \mathrm{NMR}\left(100 \mathrm{MHz}, \mathrm{CDCl}_{3}\right): \delta_{\mathrm{C}} 164.1(2 \mathrm{C}, \mathrm{CH}=\mathrm{N}), 158.0$, 140.0, 136.6, 126.7, 125.8, 117.9 (12C, Ar), 67.0 (2C, Cyclohexane), $35.0\left(6 \mathrm{C}, \mathrm{CH}_{3}\right), 34.1(6 \mathrm{C}$, $\left.\mathrm{CH}_{3}\right), 32.5$ (4C, Cyclohexane), $31.5\left(2 \mathrm{C}, \mathrm{C}\left(\mathrm{CH}_{3}\right)_{3}\right), 29.4\left(2 \mathrm{C}, \mathrm{C}\left(\mathrm{CH}_{3}\right)_{3}\right)$. IR $\left(\mathrm{KBr}, v_{\max }, \mathrm{cm}^{-1}\right)$ : $3270(\mathrm{OH})$ and $1612(\mathrm{C}=\mathrm{N})$. MS $(\mathrm{EI}): \mathrm{m} / z 547(\mathrm{M}+\mathrm{H}), 569(\mathrm{M}+\mathrm{Na})^{+}$. Anal. calcd. for $\mathrm{C}_{36} \mathrm{H}_{54} \mathrm{~N}_{2} \mathrm{O}_{2}$ : C, 79.07; H, 9.95; N, 5.12\%. Found: C, 79.11; H, 9.88; N, 5.13\%.

$\boldsymbol{N}, \boldsymbol{N}^{\prime}$-Bis-(5-nitrosalicylidene)-trans-(1,4-cyclohexylenediamine) (3e). Yellow solid, mp 342$343{ }^{\circ} \mathrm{C} .{ }^{1} \mathrm{H}$ NMR $\left(400 \mathrm{MHz}, \mathrm{DMSO}-d_{6}\right): \delta_{\mathrm{H}} 8.84(\mathrm{~s}, 2 \mathrm{H}), 8.46(\mathrm{~d}, J 3.1,2 \mathrm{H}), 8.06$ (dd, $J$ 9.6, 3.0 $\mathrm{Hz}, 2 \mathrm{H}), 6.65(\mathrm{~d}, J 9.5 \mathrm{~Hz}, 2 \mathrm{H}), 3.65(\mathrm{br}, 2 \mathrm{H}), 2.08-2.06(\mathrm{~m}, 4 \mathrm{H}), 1.75-1.70$ (m, 4H). IR 
$\left(\mathrm{KBr}, v_{\max }, \mathrm{cm}^{-1}\right): 3271(\mathrm{OH})$ and $1607(\mathrm{C}=\mathrm{N})$. MS $(\mathrm{EI}): \mathrm{m} / \mathrm{z} 411(\mathrm{M}-\mathrm{H})^{+}$. Anal. calcd. for $\mathrm{C}_{20} \mathrm{H}_{20} \mathrm{~N}_{4} \mathrm{O}_{6}$ : C, 58.25; H, 4.89; N, 13.59\%. Found: C, 58.21; H, 4.92; N, $13.55 \%$.

$\boldsymbol{N}, \boldsymbol{N}^{\prime}$-Bis-(2-hydroxynaphthylmethylene)-trans-(1,4-cyclohexylenediamine) (3f). Yellow solid, mp 276-277 ${ }^{\circ} \mathrm{C} .{ }^{1} \mathrm{H}$ NMR (400 MHz, $\mathrm{CDCl}_{3}$ ): $\delta_{\mathrm{H}} 14.80$ (s, 2H), 8.92 (d, J 5.0 Hz, 2H), $7.92(\mathrm{~d}, J 8.3 \mathrm{~Hz}, 2 \mathrm{H}), 7.72(\mathrm{~d}, J 9.3 \mathrm{~Hz}, 2 \mathrm{H}), 7.65$ (dd, J 7.9, $1.0 \mathrm{~Hz}, 2 \mathrm{H}), 7.46$ (ddd, J 8.4, 7.0, $1.3 \mathrm{~Hz}, 2 \mathrm{H}), 7.28-7.24(\mathrm{~m}, 2 \mathrm{H}), 6.97(\mathrm{~d}, J 9.3 \mathrm{~Hz}, 2 \mathrm{H}), 3.52(\mathrm{~s}, 2 \mathrm{H}), 2.22-2.20(\mathrm{~m}, 4 \mathrm{H}), 1.83$ - $1.77(\mathrm{~m}, 4 \mathrm{H}) .{ }^{13} \mathrm{C}$ NMR $\left(100 \mathrm{MHz}, \mathrm{CDCl}_{3}\right): \delta_{\mathrm{C}} 176.5(2 \mathrm{C}, \mathrm{Ar}), 158.3(2 \mathrm{C}, \mathrm{CH}=\mathrm{N}), 137.3$, 134.5, 129.3, 128.3, 125.8, 125.4, 122.7, 119.2, 106.4 (18C, Ar), 59.0, 32.0 (6C, Cyclohexane). IR $\left(\mathrm{KBr}, v_{\max }, \mathrm{cm}^{-1}\right): 3277(\mathrm{OH})$ and $1617(\mathrm{C}=\mathrm{N})$. MS $(\mathrm{EI}): \mathrm{m} / \mathrm{z} 423(\mathrm{M}+\mathrm{H})^{+}$. Anal. calcd. for $\mathrm{C}_{28} \mathrm{H}_{26} \mathrm{~N}_{2} \mathrm{O}_{2}$ : C, 79.59; H, 6.20; N, 6.63\%. Found: C, 79.62; H, 6.17; N, 6.60\%.

$\boldsymbol{N}, \boldsymbol{N}^{\prime}$-Bis-(5-fluorosalicylidene)-trans-(1,4-cyclohexylenediamine) (3g). Yellow solid, mp 222$223{ }^{\circ} \mathrm{C} .{ }^{1} \mathrm{H}$ NMR $\left(400 \mathrm{MHz}, \mathrm{CDCl}_{3}\right): \delta_{\mathrm{H}} 13.21(\mathrm{~s}, 2 \mathrm{H}), 8.36(\mathrm{~s}, 2 \mathrm{H}), 7.05-7.00(\mathrm{~m}, 2 \mathrm{H}), 6.97-$ $6.94(\mathrm{~m}, 2 \mathrm{H}), 6.92-6.89(\mathrm{~m}, 2 \mathrm{H}), 3.33-3.31$ (m, 2H), $1.99-1.95(\mathrm{~m}, 4 \mathrm{H}), 1.77-1.71(\mathrm{~m}, 4 \mathrm{H})$. ${ }^{13} \mathrm{C}$ NMR $\left(100 \mathrm{MHz}, \mathrm{CDCl}_{3}\right): \delta_{\mathrm{C}} 162.2,162.1(2 \mathrm{C}, \mathrm{CH}=\mathrm{N}), 157.2,157.1,156.5,154.2,119.3$, 119.0, 118.6, 118.5, 118.0, 117.9, 116.4, 116.1 (12C, Ar), 66.8, 32.1 (6C, Cyclohexane). ${ }^{19} \mathrm{~F}$ NMR $\left(376 \mathrm{MHz}, \mathrm{CDCl}_{3}\right) \delta$-125.91. IR $\left(\mathrm{KBr}, v_{\max }, \mathrm{cm}^{-1}\right): 3275(\mathrm{OH})$ and $1611(\mathrm{C}=\mathrm{N})$. MS (EI): $m / z 359(\mathrm{M}+\mathrm{H})^{+}$. Anal. calcd. for $\mathrm{C}_{20} \mathrm{H}_{20} \mathrm{~F}_{2} \mathrm{~N}_{2} \mathrm{O}_{2}$ : C, 67.03; H, 5.62; N, 7.82. Found: C, 67.11; $\mathrm{H}, 5.60 ; \mathrm{N}, 7.78 \%$.

$\boldsymbol{N}, \boldsymbol{N}^{\prime}$-Bis-(5-bromosalicylidene)-trans-(1,4-cyclohexylenediamine) (3h). Yellow solid, mp 266-267 ${ }^{\circ} \mathrm{C} .{ }^{1} \mathrm{H}$ NMR $\left(400 \mathrm{MHz}, \mathrm{CDCl}_{3}\right): \delta_{\mathrm{H}} 13.51(\mathrm{~s}, 2 \mathrm{H}), 8.35$ (s, 2H), 7.39-7.37 (m, 4H), 6.87 $(\mathrm{s}, 1 \mathrm{H}), 6.85(\mathrm{~s}, 1 \mathrm{H}), 3.33(\mathrm{br}, 2 \mathrm{H}), 1.99-1.98(\mathrm{~m}, 4 \mathrm{H}), 1.75-1.70(\mathrm{~m}, 4 \mathrm{H}) .{ }^{13} \mathrm{C}$ NMR $(100$ $\left.\mathrm{MHz}, \mathrm{CDCl}_{3}\right): \delta_{\mathrm{C}} 162.0(2 \mathrm{C}, \mathrm{CH}=\mathrm{N}), 160.2,134.8,133.3,120.0,119.0,110.0$ (12C, Ar), 66.7, 32.9 (6C, Cyclohexane). IR (KBr, $\left.v_{\max }, \mathrm{cm}^{-1}\right): 3277(\mathrm{OH})$ and $1612(\mathrm{C}=\mathrm{N}) . \mathrm{MS}(\mathrm{EI}): \mathrm{m} / z, 480(\mathrm{M}$ $+\mathrm{H})^{+}$. Anal. calcd. for $\mathrm{C}_{20} \mathrm{H}_{20} \mathrm{Br}_{2} \mathrm{~N}_{2} \mathrm{O}_{2}$ : C, 50.02; H, 4.20; N, 5.83. Found: C, 49.98; H, 4.22; N, $5.79 \%$.

1,4-Bis-[(2-hydroxybenzylideneamino)methyl]benzene (4a). ${ }^{42}$ Yellow solid, mp 140-142 ${ }^{\circ} \mathrm{C}$. ${ }^{1} \mathrm{H}$ NMR (400 MHz, $\mathrm{CDCl}_{3}$ ): $\delta_{\mathrm{H}} 13.39$ (s, 2H), 8.43 (s, 2H), 7.33 (dt, J 3.2, $1.9 \mathrm{~Hz}, 2 \mathrm{H}$ ), 7.30 (s, 4H), 7.28 (dd, J 7.6, 1.6 Hz, 2H), 6.98 (d, J 8.3 Hz, 2H), 6.89 (td, J 7.5, $1.1 \mathrm{~Hz}, 2 \mathrm{H}), 4.79$ (d, $J$ $1.0 \mathrm{~Hz}, 4 \mathrm{H}) .{ }^{13} \mathrm{C} \mathrm{NMR}\left(400 \mathrm{MHz}, \mathrm{CDCl}_{3}\right): \delta_{\mathrm{C}} 165.7(2 \mathrm{C}, \mathrm{CH}=\mathrm{N}), 161.1,137.3,132.4,131.5$, 128.1, 118.8, 118.6, 117.0 (18C, Ar), $62.8\left(2 \mathrm{C}, \mathrm{CH}_{2}\right)$. IR (KBr, $\left.v_{\max }, \mathrm{cm}^{-1}\right): 3281(\mathrm{OH})$ and 1611 $(\mathrm{C}=\mathrm{N})$. MS (EI): $m / z 345(\mathrm{M}+\mathrm{H})^{+}, 367(\mathrm{M}+\mathrm{Na})^{+}$. Anal. calcd. for $\mathrm{C}_{22} \mathrm{H}_{20} \mathrm{~N}_{2} \mathrm{O}_{2}: \mathrm{C}, 76.72 ; \mathrm{H}$, 5.85 ; N, 8.13. Found: C, 76.69; H, 5.90; N, 8.10\%.

1,4-Bis-[(5-methyl-2-hydroxybenzylideneamino)methyl]benzene (4b). Yellow solid, mp 154$155{ }^{\circ} \mathrm{C} .{ }^{1} \mathrm{H}$ NMR $\left(400 \mathrm{MHz}, \mathrm{CDCl}_{3}\right) \delta_{\mathrm{H}} 13.12$ (s, 2H), 8.37 (s, 2H), 7.29 (s, 4H), 7.12 (d, J 8.3 $\mathrm{Hz}, 2 \mathrm{H}), 7.06$ (s, 2H), $6.88(\mathrm{~d}, J 8.3 \mathrm{~Hz}, 2 \mathrm{H}), 4.78$ (s, 4H), 2.29 (s, 6H). ${ }^{13} \mathrm{C} \mathrm{NMR}(100 \mathrm{MHz}$, $\left.\mathrm{CDCl}_{3}\right): \delta_{\mathrm{C}} 165.7(2 \mathrm{C}, \mathrm{CH}=\mathrm{N}), 158.8,137.4,133.2,131.5,128.1,127.6,118.5,116.7$ (18C, Ar), $62.9\left(2 \mathrm{C}, \mathrm{CH}_{2}\right), 20.3\left(2 \mathrm{C}, \mathrm{CH}_{3}\right)$. IR $\left(\mathrm{KBr}, v_{\max }, \mathrm{cm}^{-1}\right): 3271(\mathrm{OH})$ and $1622(\mathrm{C}=\mathrm{N})$. MS (EI): $\mathrm{m} / z$ $373(\mathrm{M}+\mathrm{H})^{+}, 395(\mathrm{M}+\mathrm{Na})^{+}$. Anal. calcd. for $\mathrm{C}_{24} \mathrm{H}_{24} \mathrm{~N}_{2} \mathrm{O}_{2}$ : C, 77.39; $\mathrm{H}, 6.49 ; \mathrm{N}, 7.52$. Found: $\mathrm{C}, 77.41 ; \mathrm{H}, 6.46 ; \mathrm{N}, 7.50 \%$. 
1,4-Bis-[(5-methoxy-2-hydroxybenzylideneamino)methyl]benzene (4c). Yellow solid, mp 150-151 ${ }^{\circ} \mathrm{C} .{ }^{1} \mathrm{H}$ NMR $\left(400 \mathrm{MHz}, \mathrm{CDCl}_{3}\right): \delta_{\mathrm{H}} 12.77$ (s, 2H), 8.66 (s, 2H), 7.33 (s, 4H), 7.08 (d, J $3.1 \mathrm{~Hz}, 2 \mathrm{H}), 6.96(\mathrm{dd}, J$ 8.9, $3.1 \mathrm{~Hz}, 2 \mathrm{H}), 6.83(\mathrm{~d}, J 8.9 \mathrm{~Hz}, 2 \mathrm{H}), 4.79(\mathrm{~s}, 4 \mathrm{H}), 3.72(\mathrm{~s}, 6 \mathrm{H}) .{ }^{13} \mathrm{C}$ NMR $\left(100 \mathrm{MHz}, \mathrm{CDCl}_{3}\right): \delta_{\mathrm{C}} 166.5(2 \mathrm{C}, \mathrm{CH}=\mathrm{N}), 154.8,152.0,138.0,128.5,119.8,119.0,117.6$, 115.3 (18C, Ar), $62.4\left(2 \mathrm{C}, \mathrm{CH}_{2}\right), 55.9\left(2 \mathrm{C}, \mathrm{CH}_{3}\right)$. IR $\left(\mathrm{KBr}, v_{\max }, \mathrm{cm}^{-1}\right): 3280(\mathrm{OH})$ and 1621 $(\mathrm{C}=\mathrm{N})$. MS (EI): $m / z 405(\mathrm{M}+\mathrm{H})^{+}, 427(\mathrm{M}+\mathrm{Na})^{+}$. Anal. calcd. for $\mathrm{C}_{24} \mathrm{H}_{24} \mathrm{~N}_{2} \mathrm{O}_{4}: \mathrm{C}, 71.27 ; \mathrm{H}$, 5.98; N, 6.93. Found: C, 71.30; H, 6.01; N, 6.89\%.

1,4-Bis-[(4-methoxy-2-hydroxybenzylideneamino)methyl]benzene (4d). Yellow solid, mp 132-133 ${ }^{\circ} \mathrm{C} .{ }^{1} \mathrm{H}$ NMR $\left(400 \mathrm{MHz}, \mathrm{CDCl}_{3}\right): \delta_{\mathrm{H}} 13.86$ (s, 2H), 8.27 (s, 2H), 7.28 (s, 4H), 7.12 (d, J $4.3 \mathrm{~Hz}, 2 \mathrm{H}), 6.44(\mathrm{~d}, J 2.4 \mathrm{~Hz}, 2 \mathrm{H}), 6.41$ (d, J $2.4 \mathrm{~Hz}, 1 \mathrm{H}), 6.39$ (d, J $2.4 \mathrm{~Hz}, 1 \mathrm{H}), 4.73$ (s, 4H), $3.80(\mathrm{~s}, 6 \mathrm{H}) .{ }^{13} \mathrm{C} \mathrm{NMR}\left(100 \mathrm{MHz}, \mathrm{CDCl}_{3}\right): \delta_{\mathrm{C}} 164.9,164.6(2 \mathrm{C}, \mathrm{CH}=\mathrm{N}), 163.5,137.4,132.7$, 128.0, 112.4, 106.4, 101.2 (18C, Ar), $61.7\left(2 \mathrm{C}, \mathrm{CH}_{2}\right), 55.3\left(2 \mathrm{C}, \mathrm{CH}_{3}\right) . \mathrm{IR}\left(\mathrm{KBr}, v_{\max }, \mathrm{cm}^{-1}\right): 3277$ $(\mathrm{OH})$ and $1623(\mathrm{C}=\mathrm{N})$. $\mathrm{MS}(\mathrm{EI}): m / z 405(\mathrm{M}+\mathrm{H})^{+}, 427(\mathrm{M}+\mathrm{Na})^{+}$. Anal. calcd. for $\mathrm{C}_{24} \mathrm{H}_{24} \mathrm{~N}_{2} \mathrm{O}_{4}$ : C, 71.27; H, 5.98; N, 6.93. Found: C, 71.28; H, 5.95; N, 6.99\%.

1,4-Bis-[(3,5-di-tert-butyl-2-hydroxybenzylideneamino)methyl]benzene (4e). ${ }^{43}$ Yellow solid, mp 183-184 ${ }^{\circ} \mathrm{C} .{ }^{1} \mathrm{H}$ NMR $\left(400 \mathrm{MHz}, \mathrm{CDCl}_{3}\right.$ ): $\delta_{\mathrm{H}} 13.72$ (s, 2H), 8.46 (s, 2H), 7.40 (d, J $2.5 \mathrm{~Hz}$, 2H), $7.32(\mathrm{~s}, 4 \mathrm{H}), 7.11(\mathrm{~d}, J 2.5 \mathrm{~Hz}, 2 \mathrm{H}), 4.79(\mathrm{~s}, 4 \mathrm{H}), 1.44(\mathrm{~s}, 18 \mathrm{H}), 1.32(\mathrm{~s}, 18 \mathrm{H}) .{ }^{13} \mathrm{C} \mathrm{NMR}$ $\left(100 \mathrm{MHz}, \mathrm{CDCl}_{3}\right): \delta_{\mathrm{C}} 166.7(2 \mathrm{C}, \mathrm{CH}=\mathrm{N}), 158.0,140.0,137.4,136.7,128.2,127.0,126.0,117.9$ (18C, Ar), $62.9\left(2 \mathrm{C}, \mathrm{CH}_{2}\right), 35.0\left(6 \mathrm{C}, \mathrm{CH}_{3}\right), 34.1\left(6 \mathrm{C}, \mathrm{CH}_{3}\right), 31.5\left(2 \mathrm{C}, \mathrm{C}\left(\mathrm{CH}_{3}\right)_{3}\right), 29.4(2 \mathrm{C}$, $\left.C\left(\mathrm{CH}_{3}\right)_{3}\right)$. IR $\left(\mathrm{KBr}, v_{\max }, \mathrm{cm}^{-1}\right): 3287(\mathrm{OH})$ and $1617(\mathrm{C}=\mathrm{N})$. MS $(\mathrm{EI}): m / z, 591(\mathrm{M}+\mathrm{Na})^{+}$. Anal. calcd. for $\mathrm{C}_{38} \mathrm{H}_{52} \mathrm{~N}_{2} \mathrm{O}_{2}$ : C, 80.24; H, 9.21; N, 4.92. Found: C, 80.19; H, 9.27; N, 5.01\%.

1,4-Bis-[(5-nitro-2-hydroxybenzylideneamino)methyl]benzene (4f). Yellow solid, mp 275$276{ }^{\circ} \mathrm{C} .{ }^{1} \mathrm{H}$ NMR $\left(400 \mathrm{MHz}, \mathrm{CDCl}_{3}\right): \delta_{\mathrm{H}} 13.35$ (s, 2H), 8.43 (s, 2H), 7.33-7.26 (m, 6H), 6.97$6.95(\mathrm{~m}, 2 \mathrm{H}), 6.88(\mathrm{td}, J 7.5,1.1 \mathrm{~Hz}, 2 \mathrm{H}), 4.80(\mathrm{~d}, J 1.1 \mathrm{~Hz}, 4 \mathrm{H}) .{ }^{13} \mathrm{C} \mathrm{NMR}\left(400 \mathrm{MHz}, \mathrm{CDCl}_{3}\right)$ : $\delta_{\mathrm{C}} 165.6(2 \mathrm{C}, \mathrm{CH}=\mathrm{N}), 161.1,137.3,132.3,131.4,128.1,118.8,118.6,117.0(18 \mathrm{C}, \mathrm{Ar}), 62.8$ $\left(2 \mathrm{C}, \mathrm{CH}_{2}\right)$. IR $\left(\mathrm{KBr}, v_{\max }, \mathrm{cm}^{-1}\right): 3270(\mathrm{OH})$ and $1621(\mathrm{C}=\mathrm{N})$. MS (EI): $m / z 433(\mathrm{M}-\mathrm{H})^{+}$. Anal. calcd. for $\mathrm{C}_{22} \mathrm{H}_{18} \mathrm{~N}_{4} \mathrm{O}_{6}$ : C, 60.83; H, 4.18; N, 12.90. Found: C, 60.80; H, 4.20; N, 12.97\%.

1,4-Bis-[(2-hydroxynaphthylmethyleneamino)methyl]benzene (4g). Yellow solid, mp 263$264{ }^{\circ} \mathrm{C} .{ }^{1} \mathrm{H}$ NMR $\left(400 \mathrm{MHz}, \mathrm{DMSO}-d_{6}\right): \delta_{\mathrm{H}} 14.36$ (s, 2H), 9.30 (s, 2H), 8.10 (s, 2H), 7.73 (s, $3 \mathrm{H}), 7.64(\mathrm{~s}, 3 \mathrm{H}), 7.44(\mathrm{~s}, 4 \mathrm{H}), 7.20(\mathrm{~s}, 2 \mathrm{H}), 6.73(\mathrm{~s}, 2 \mathrm{H}), 4.87(\mathrm{~s}, 4 \mathrm{H})$. IR $\left(\mathrm{KBr}, v_{\max }, \mathrm{cm}^{-1}\right): 3276$ $(\mathrm{OH})$ and $1622(\mathrm{C}=\mathrm{N})$. $\mathrm{MS}(\mathrm{EI}): \mathrm{m} / z 445(\mathrm{M}+\mathrm{H})^{+}, 467(\mathrm{M}+\mathrm{Na})^{+}$. Anal. calcd. for $\mathrm{C}_{30} \mathrm{H}_{24} \mathrm{~N}_{2} \mathrm{O}_{2}$ : C, 81.06; H, 5.44; N, 6.30. Found: C, 81.10; H, 5.40; N, 6.26\%.

1,4-Bis-[(5-fluoro-2-hydroxybenzylideneamino)methyl]benzene (4h). Yellow solid, mp 183$184{ }^{\circ} \mathrm{C} .{ }^{1} \mathrm{H} \mathrm{NMR}\left(400 \mathrm{MHz}, \mathrm{CDCl}_{3}\right): \delta_{\mathrm{H}} 13.05$ (s, 2H), 8.37 (s, 2H), $7.30(\mathrm{~s}, 4 \mathrm{H})$, 7.05-6.90 (m, $6 \mathrm{H}), 4.81(\mathrm{~s}, 4 \mathrm{H}) .{ }^{13} \mathrm{C} \mathrm{NMR}\left(100 \mathrm{MHz}, \mathrm{CDCl}_{3}\right): \delta_{\mathrm{C}} 164.6(2 \mathrm{C}, \mathrm{CH}=\mathrm{N}), 157.1,154.2,137.1$, 128.2, 119.5, 119.3, 118.5, 118.1, 118.0, 116.6, $116.3(18 \mathrm{C}, \mathrm{Ar}), 62.9\left(2 \mathrm{C}, \mathrm{CH}_{2}\right) .{ }^{19} \mathrm{~F}$ NMR $(376$ $\left.\mathrm{MHz} \mathrm{CDCl}_{3}\right) \delta$-125.90. IR (KBr, $\left.v_{\max }, \mathrm{cm}^{-1}\right): 3275(\mathrm{OH})$ and $1623(\mathrm{C}=\mathrm{N})$. MS (EI): $\mathrm{m} / z, 381(\mathrm{M}$ $+\mathrm{H})^{+}, 403(\mathrm{M}+\mathrm{Na})^{+}$. Anal. calcd. for $\mathrm{C}_{22} \mathrm{H}_{18} \mathrm{~F}_{2} \mathrm{~N}_{2} \mathrm{O}_{2}$ : C, 69.46; H, 4.77; N, 7.36. Found: C, $69.43 ; \mathrm{H}, 4.79 ; \mathrm{N}, 7.30 \%$. 
1,4-Bis-[(5-bromo-2-hydroxybenzylideneamino)methyl]benzene (4i). Yellow solid, mp 199$201{ }^{\circ} \mathrm{C} .{ }^{1} \mathrm{H}$ NMR $\left(400 \mathrm{MHz}, \mathrm{CDCl}_{3}\right.$ ): $\delta_{\mathrm{H}} 13.35$ (s, 2H), 8.35 (s, 2H), 7.38 (s, 4H), 7.29 (s, 4H), $6.85(\mathrm{~d}, J 8.5 \mathrm{~Hz}, 2 \mathrm{H}), 4.81(\mathrm{~s}, 4 \mathrm{H}) .{ }^{13} \mathrm{C} \mathrm{NMR}\left(100 \mathrm{MHz}, \mathrm{CDCl}_{3}\right): \delta_{\mathrm{C}} 164.3(2 \mathrm{C}, \mathrm{CH}=\mathrm{N}), 160.1$, 137.0, 135.0, 133.5, 128.2, 120.1, 119.0, 110.0 (18C, Ar), $62.7\left(2 \mathrm{C}, \mathrm{CH}_{2}\right) . \mathrm{IR}\left(\mathrm{KBr}, v_{\max }, \mathrm{cm}^{-1}\right)$ : $3288(\mathrm{OH})$ and $1619(\mathrm{C}=\mathrm{N})$. MS $(\mathrm{EI}): m / z 502(\mathrm{M}+\mathrm{H})^{+}, 524(\mathrm{M}+\mathrm{Na})^{+}$. Anal. calcd. for $\mathrm{C}_{22} \mathrm{H}_{18} \mathrm{Br}_{2} \mathrm{~N}_{2} \mathrm{O}_{2}$ : C, 52.62; H, 3.61; N, 5.58. Found: C, 52.60; H, 3.69; N, 5.61\%.

$N, N^{\prime}$-Bis-(4-methoxysalicylidene)-1,2-ethylenediamine (5). ${ }^{44}$ Yellow solid, mp $160-162{ }^{\circ} \mathrm{C} .{ }^{1} \mathrm{H}$ NMR (400 MHz, CDCl $): \delta_{\mathrm{H}} 13.69$ (s, 2H), 8.18 (s, 2H), 7.07 (d, J 8.5 Hz, 2H), 6.41 (d, J 2.4 $\mathrm{Hz}, 2 \mathrm{H}), 6.37(\mathrm{dd}, J 8.5,2.4 \mathrm{~Hz}, 2 \mathrm{H}), 3.82(\mathrm{~s}, 4 \mathrm{H}), 3.77(\mathrm{~s}, 6 \mathrm{H}) .{ }^{13} \mathrm{C} \mathrm{NMR}\left(400 \mathrm{MHz}, \mathrm{CDCl}_{3}\right): \delta_{\mathrm{C}}$ $165.4(2 \mathrm{C}, \mathrm{CH}=\mathrm{N}), 164.7,163.5,132.7,112.3,106.4,101.1(12 \mathrm{C}, \mathrm{Ar}), 58.7\left(2 \mathrm{C}, \mathrm{CH}_{2}\right), 55.3(2 \mathrm{C}$, $\left.\mathrm{CH}_{3}\right)$. IR $\left(\mathrm{KBr}, v_{\max }, \mathrm{cm}^{-1}\right)$ : $3264(\mathrm{OH})$ and $1611(\mathrm{C}=\mathrm{N})$. Anal. calcd. for $\mathrm{C}_{18} \mathrm{H}_{20} \mathrm{~N}_{2} \mathrm{O}_{4}: \mathrm{C}, 65.84$; H, 6.14; N, 8.53. Found: C, 65.79; H, 6.19; N, 8.57\%.

Synthesis of 4'-[4"'-(3"'-formyl-4"'-hydroxyphenyl)-1H-1",2", 3"'-triazol-1"'-yl]-2,2'-bipyridine $N^{\prime}$-oxide (8). A solution of 6 (10 mmol, $\left.1.46 \mathrm{~g}\right)$ and 7 (10 mmol, 2.13) in $t$ - $\mathrm{BuOH}: \mathrm{H}_{2} \mathrm{O}$ (4: $1,25 \mathrm{~mL}$ ) in the presence of $15 \mathrm{~mol} \% \mathrm{CuSO}_{4} \cdot 5 \mathrm{H}_{2} \mathrm{O}$ with $30 \mathrm{~mol} \%$ sodium ascorbate was stirred at $90{ }^{\circ} \mathrm{C}$ for $36 \mathrm{~h}$. The reaction was monitored by TLC following the disappearance of $\mathbf{6}$ and the generation of the triazole derivative. The mixture was heated overnight at $60{ }^{\circ} \mathrm{C}$. The still hot mixture was filtered through Celite followed by washing with $\mathrm{MeOH}(3 \times 10 \mathrm{~mL})$. The combined filtrate and washings were evaporated under reduced pressure. The desired product was obtained in $97.5 \%$ yield, mp 204-205 ${ }^{\circ} \mathrm{C} .{ }^{1} \mathrm{H}$ NMR $\left(400 \mathrm{MHz}, \mathrm{CDCl}_{3}\right): \delta_{\mathrm{H}} 11.13(\mathrm{~s}, 1 \mathrm{H})$, 10.02 (s, $1 \mathrm{H}), 9.14$ (d, $J 8.18 \mathrm{~Hz}, 1 \mathrm{H}), 8.78-8.77$ (m, $1 \mathrm{H}), 8.68$ (d, J $3.2 \mathrm{~Hz}, 1 \mathrm{H}), 8.46$ (d, $J$ $7.2 \mathrm{~Hz}, 1 \mathrm{H}), 8.34(\mathrm{~s}, 1 \mathrm{H}), 8.22(\mathrm{~d}, J 2.1 \mathrm{~Hz}, 1 \mathrm{H}), 8.01$ (dd, J 8.6, 2.2 Hz, $1 \mathrm{H}), 7.96$ (dd, J 7.2, $3.3 \mathrm{~Hz}, 1 \mathrm{H}), 7.90$ (ddd, $J$ 9.6, 7.8, $1.9 \mathrm{~Hz}, 1 \mathrm{H}), 7.45-7.42(\mathrm{~m}, 1 \mathrm{H}), 7.12 \mathrm{ppm}(\mathrm{d}, J 8.7 \mathrm{~Hz}, 1 \mathrm{H})$. ${ }^{13} \mathrm{C}$ NMR $\left(100 \mathrm{MHz}, \mathrm{CDCl}_{3}\right): \delta_{\mathrm{C}} 190.7$ (1C, CHO), 160.9, 149.5, 148.6, 147.0, 142.1, 136.6, $133.1,132.3,125.3,125.1,125.0,122.7,121.3,118.9,118.1,116.9,116.3$ (18C, Ar). MS (EI): $m / z 358(\mathrm{M}-\mathrm{H})^{+}, 382(\mathrm{M}+\mathrm{Na})^{+}$. Anal. calcd. for $\mathrm{C}_{19} \mathrm{H}_{13} \mathrm{~N}_{5} \mathrm{O}_{3}: \mathrm{C}, 63.51 ; \mathrm{H}, 3.65 ; \mathrm{N}, 19.49$. Found: C, 63.48; H, 3.69; N, 19.53\%.

\section{Synthesis of bis-Schiff bases 9a-c}

These compounds were prepared by condensation of $\mathbf{8}$ with $\mathbf{2 a - c}$ using the procedures described for the synthesis of compounds 3-5 (see Table 2).

9a. Yellow solid, mp 177-179 ${ }^{\circ} \mathrm{C}$. ${ }^{1} \mathrm{H}$ NMR (400 MHz, DMSO- $d_{6}$ ): $\delta_{\mathrm{H}} 13.92$ (s, 2H), 9.44 (s, 2H), 8.87-8.62 (m, 9H), $8.31(\mathrm{~s}, 2 \mathrm{H}), 8.09-8.01(\mathrm{~m}, 4 \mathrm{H}), 7.88(\mathrm{~s}, 2 \mathrm{H}), 7.57$ (s, 3H), 7.03 (br, 2H), 4.09 (br, 2H), 2.08 (br, 4H), 1.70 (br, 4H). IR (KBr, $\left.v_{\max }, \mathrm{cm}^{-1}\right): 3281(\mathrm{OH})$ and $1616(\mathrm{C}=\mathrm{N})$. MS (EI): $m / z 819(\mathrm{M}+\mathrm{Na})^{+}$. Anal. calcd. for $\mathrm{C}_{44} \mathrm{H}_{36} \mathrm{~N}_{12} \mathrm{O}_{4}: \mathrm{C}, 66.32 ; \mathrm{H}, 4.55 ; \mathrm{N}, 21.09$. Found: $\mathrm{C}$, 66.28; H, 4.60; N, 21.13\%.

9b. Yellow solid, mp 260-261 ${ }^{\circ} \mathrm{C} .{ }^{1} \mathrm{H}$ NMR (400 MHz, DMSO- $\left.d_{6}\right): \delta_{\mathrm{H}} 9.43$ (s, 2H), 8.80-8.60 $(\mathrm{m}, 10 \mathrm{H}), 8.08-7.88(\mathrm{~m}, 9 \mathrm{H}), 7.56-7.13(\mathrm{~m}, 5 \mathrm{H}), 7.01(\mathrm{~s}, 2 \mathrm{H}), 4.86(\mathrm{~s}, 4 \mathrm{H}) . \mathrm{IR}\left(\mathrm{KBr}, v_{\max }, \mathrm{cm}^{-1}\right)$ : $(\mathrm{KBr}) 3288(\mathrm{OH})$ and $1631(\mathrm{C}=\mathrm{N})$. MS $(\mathrm{EI}): \mathrm{m} / z 841(\mathrm{M}+\mathrm{Na})^{+}$. Anal. calcd. for $\mathrm{C}_{46} \mathrm{H}_{34} \mathrm{~N}_{12} \mathrm{O}_{4}$ : C, 67.47; H, 4.19; N, 20.53. Found: C, 67.51; H, 4.10; N, 20.48\%. 
9c. Yellow solid, mp 166-168 ${ }^{\circ} \mathrm{C} .{ }^{1} \mathrm{H}$ NMR (400 MHz, DMSO- $\left.d_{6}\right): \delta_{\mathrm{H}} 13.70$ (s, 2H), 9.42 (s, $2 \mathrm{H}), 8.86-8.60(\mathrm{~m}, 10 \mathrm{H}), 8.06$ (br, 6H), 7.87 (s, 2H), 7.56 (s, 2H), 7.01 (s, 2H), 4.01 (s, 4H). IR $\left(\mathrm{KBr}, v_{\max }, \mathrm{cm}^{-1}\right): 3264(\mathrm{OH})$ and $1611(\mathrm{C}=\mathrm{N})$. MS $(\mathrm{EI}): \mathrm{m} / \mathrm{z} 741(\mathrm{M}-\mathrm{H})^{+}$. Anal. calcd. for $\mathrm{C}_{40} \mathrm{H}_{30} \mathrm{~N}_{12} \mathrm{O}_{4}$ : C, 64.68; H, 4.07; N, 22.63. Found: C, 64.60; H, 4.11; N, 22.66\%.

\section{Supplementary Material}

The NMR and MS spectra of compounds can be found in the Supplementary Material section of this article.

\section{Acknowledgements}

We are very thankful to the Department of Organic Chemistry, Arrhenius Lab., Stockholm University for providing laboratory facilities.

\section{References}

1. Mandewale, M.; Thorat, C. B.; Patil, U.; Yamgar, R. Int. J. Chem. Phys. Sci. 2015, 3, 1919.

2. Qin, W.; Long, S.; Panunzio, M.; Biondi, S. Molecules 2013, 18, 12264. http://dx.doi.org/10.3390/molecules181113636

3. Murtaza, G.; Mumtaz, A.; Khan, F. A.; Ahmad, S.; Azhar, S.; Najam-Ul-Haq, M.; Atif, M.; Khan, S. A.; Maalik, A.; Alam, F.; Hussain, I. Acta Pol. Pharm. Drug Res. 2014, 71, 531.

4. Blagus, A.; Cinčić, D.; Friščić, T.; Kaitner, B.; Stilinović, V. Maced. J. Chem. Chem. Eng. 2010, 29, 117.

5. Raghuwanshi, P. B.; Mahalle, P. V. Der Pharma Chemica 2014, 6, 262.

6. Maity, S.; Khan, S. A.; Ahmad, S. Int. J. Pharm. Bio. Sci. 2012, 3, 90.

7. Anacona, J. R.; Noriega, N.; Camus, J. Spectrochim. Acta A 2015, 137, 16. http://dx.doi.org/10.1016/j.saa.2014.07.091

PMid:25194315

8. Chigurupati, S.; Designing, S. J. Med. Biol. Eng. 2015, 4, 363.

9. Muthumani, P.; Meera, R.; Venkatraman, S.; Murugan, P. D. J. Chem. Pharm. Res. 2010, 2, 433.

10. Harpstrite, S. E.; Collins, S. D.; Oksman, A.; Goldberg, D. E.; Sharma, V. Med. Chem. 2008, 4, 392 .

http://dx.doi.org/10.2174/157340608784872280

PMid:18673153 
11. Aziz, A. N.; Taha, M.; Ismail, N. H.; Anouar, E. H.; Yousuf, S.; Jamil, W.; Awang, K.; Ahmat, N.; Khan, K. M.; Kashif, S. M. Molecules 2014, 19, 8414.

http://dx.doi.org/10.3390/molecules19068414

PMid:24950444

12. Cheng, L.; Tang, J.; Luo, H.; Jin, X.; Dai, F.; Yang, J.; Qian, Y.; Li, X.; Zhou, B. Bioorg. Med. Chem. Lett. 2010, 20, 2417.

http://dx.doi.org/10.1016/j.bmcl.2010.03.039

13. Jarrahpour, A.; Khalili, D.; Clercq, E.; Salmi, C.; Brunel, J. M. Molecules 2007, 12, 1720. http://dx.doi.org/10.3390/12081720

PMid:17960083

14. Kalaivani, S.; Priya, N. P.; Arunachalam, S. Int. J. App. Bio. Pharm. Tech. 2012, 3, 219.

15. Liang, C.; Xia, J.; Lei, D.; Li, X.; Yao, Q.; Gao, J. Eur. J. Med. Chem. 2013, 4, 742.

16. Chinnasamy, R.; Sundararagan, P. R.; Govindaraj, S. J. Adv. Pharm. Technol. Res. 2010, 1, 342.

http://dx.doi.org/10.4103/0110-5558.72428

PMid:22247869

17. Jain, J. S.; Srivastava, R. S.; Aggarwal, N.; Sinha, R. Cent. Nerv. Syst. Agents, Med. Chem. 2007, 7, 200.

http://dx.doi.org/10.2174/187152407781669143

18. Varma, M.; Pandeya, S., N.; Singh, K. N.; Stables, J. P. Acta Pharm. 2004, 54, 49.

19. Aslam, M. A. S.; Mahmood, S. U.; Shahid, M.; Saeed, A.; Iqbal, J. Eur. J. Med. Chem. 2011, $46,5473$.

http://dx.doi.org/10.1016/j.ejmech.2011.09.009

PMid:21981981

20. Chazin, E. L.; Sanches, P. S.; Lindgren, E. B.; Júnior, W. T. V.; Pinto, L. C.; Burbano, R. M. R.; Yoneda, J. D.; Leal, K. Z.; Gomes, C. R. B.; Wardell, J. L.; Wardell, S. M. S.; Montenegro, R. C.; Vasconcelos, T. R. A. Molecules 2015, 20, 1968.

http://dx.doi.org/10.3390/molecules20021968

PMid:25633329

21. Dhanaraj, C. J.; Nair, M. S. J. Coord. Chem. 2009, 62, 4018.

http://dx.doi.org/10.1080/00958970903191142

22. Iqbal, A.; Siddiqui, H. L.; Ashraf, C. M.; Ahmad, M.; Weaver, G. W. Molecules 2007, 12, 245.

http://dx.doi.org/10.3390/12020245

PMid:17846575

23. Sobczyk, L.; Grabowski, S. J.; Krygowski, T. M. Chem. Rev. 2005, 105, 3513. http://dx.doi.org/10.1021/cr030083c

PMid:16218560

24. Szady, C. A.; Grech, E.; Rozwadowski, Z.; Dziembowska, T.; Schilf, W.; Kamienski, B. J. Mol. Struct. 2001, 565, 125. 
http://dx.doi.org/10.1016/S0022-2860(00)00788-2

25. Schilf, W.; Kamienski, B.; Dziembowska, T. J. Mol. Struct. 2002, 602, 41. http://dx.doi.org/10.1016/S0022-2860(01)00742-6

26. Lehn, J. M. Supramolecular Chemistry: Concepts and perspectives, VCH: Weinheim, 1995. http://dx.doi.org/10.1002/3527607439

27. Liang, C.; Xia, J.; Lei, D.; Li, X.; Yao, Q.; Gao, J. Eur. J. Med. Chem. 2014, 74, 742. http://dx.doi.org/10.1016/j.ejmech.2013.04.040 PMid:24176732

28. Fang, Z.; Cao, C.; Chen, J.; Deng, X. J. Mol. Struct. 2014, 1063, 307. http://dx.doi.org/10.1016/j.molstruc.2014.01.073

29. Zhao, J.; Zhao, B.; Liu, J.; Xu, W.; Wang, Z. Spectrochim. Acta A 2001, 57, 149. http://dx.doi.org/10.1016/S1386-1425(00)00353-X

30. Minkin, V. I.; Tsukanov, A. V.; Dubonosov, A. D.; Bren, V. A. J. Mol. Struct. 2011, 998, 179. http://dx.doi.org/10.1016/j.molstruc.2011.05.029

31. Iwan, A.; Janeczek, H.; Jarzabek, B.; Rannou, P. Materials 2009, 2, 38. http://dx.doi.org/10.3390/ma2010038

32. Iwan, A.; Sek, D. Prog. Polym. Sci. 2008, 33, 289. http://dx.doi.org/10.1016/j.progpolymsci.2007.09.005

33. Youn, J.; Kewalramani, S.; Emery, J. D.; Shi, Y.; Zhang, S.; Chang, H.-C.; Liang, Y.-J.; Yeh, C.-M.; Feng, C.-Y.; Huang, H.; Stern, C.; Chen, L.-H.; Ho, J.-C.; Chen, M.-C.; Bedzyk, M. J.; Facchetti, A.; Marks, T. J. Adv. Functional Mat. 2013, 23, 3850. http://dx.doi.org/10.1002/adfm.201203439

34. Khan, K. M.; Jamil, W.; Ambreen, N.; Taha, M.; Perveen, S.; Morales, G. A. Ultrason. Sonochem. 2014, 21, 1200.

http://dx.doi.org/10.1016/j.ultsonch.2013.12.011

PMid:24398059

35. Singh, B. S.; Lobo, H. R.; Pinjari, D. V.; Jarag, K. J.; Pandit, A. B.; Shankarling, G. S. Ultrason. Sonochem. 2013, 20, 633.

http://dx.doi.org/10.1016/j.ultsonch.2012.06.003

http://dx.doi.org/10.1016/j.ultsonch.2012.09.002

PMid:23062955

36. Shaker, R. M. Arkivoc 2012, (i), 1.

37. Shaker, R. M. Heteroatom Chem. 2005, 16, 507. http://dx.doi.org/10.1002/hc.20150

38. Hussein, M. A.; Shaker, R. M.; Ameen, M. A.; Mohammed, M. F. Arch. Pharmacal Res. 2011, 34, 1239.

http://dx.doi.org/10.1007/s12272-011-0802-z

PMid:21910044 
39. Mohamed, A. M.; Al-Qalawi, H. R. M.; El-Sayed, W. A.; Arafa, W. A. A.; Alhumaimess, M. S.; Hassan, A. K. Acta Pol. Pharm. Drug Res. 2015, 72, 307.

40. Arafa, W. A. A. J. Heterocyclic Chem. 2010, 47, 1109. http://dx.doi.org/10.1002/jhet.431

41. Hancock, S. L.; Mahon, M. F.; Jones, M. D. New J. Chem. 2013, 37, 1996. http://dx.doi.org/10.1039/c3nj00111c

42. Kim, S. M.; Kim, J. -S.; Shin, D. -M.; Kim, Y. K.; Ha, Y. Bull. Korean Chem. Soc. 2001, 22 , 743.

43. Tooke, D. M.; Song, Y.; van Albada, G. A.; Reedijk, J.; Spek, A. L. Acta Cryst. 2004, 60, 1907.

44. Hariharan, P. S.; Savarimuthu, P. A. SPECTROCHIM ACTA A 2015, 136, 1658. http://dx.doi.org/10.1016/j.saa.2014.10.061

PMid:25459729

45. Safaei-Ghomi, J.; Masoomi, R. Sci. Iran. 2015, 22, 894.

46. Cinarli, A.; Gürbüz, D.; Tavman, A.; Birteksöz, A. S. Bull. Chem. Soc. Ethiop. 2011, 25 , 407.

http://dx.doi.org/10.4314/bcse.v25i3.68593

47. Beyer, C.; Wagenknecht, H. -A. J. Org. Chem. 2010, 75, 2752.

http://dx.doi.org/10.1021/jo100309r

48. Baron, A.; Herrero, C.; Quaranta, A.; Charlot, M. F.; Leibl, W.; Vauzeilles, B.; Aukauloo, A. Chem. Commun. 2011, 47, 11011.

http://dx.doi.org/10.1039/c1cc13683f 